\title{
A novel graphene pressure sensor with zig-zag shaped piezoresistors for maximum strain coverage for enhancing the sensitivity of the pressure sensor
}

\author{
Meetu Nag ${ }^{1, *}$, Ajay Kumar², and Bhanu Pratap ${ }^{3}$ (D) \\ ${ }^{1}$ Department of Electrical Engineering, Vivekananda Global University, Jaipur, India \\ 2 Department of Mechatronics Engineering, Manipal University Jaipur, Jaipur, India \\ ${ }^{3}$ Department of Mechanical Engineering, Manipal University, Jaipur, India
}

Received: 12 April 2021 / Accepted: 20 July 2021

\begin{abstract}
The demand for flexible and wearable sensors is increasing day by day due to varied applications in the biomedical field. Especially highly sensitive sensors are required for the detection of the low signal from the body. It is important to develop a pressure sensor that can convert the maximum input signal into the electrical output. In this paper, the design and performance of graphene piezoresistive pressure sensors have been investigated by zig-zag piezoresistors on the square diaphragm. On the applied pressure, deformation is sensed by the piezoresistors above the diaphragm. Finite element analysis is carried out to investigate the effect of zig-zag piezoresistors on the square diaphragm. Simulated results for the optimized design are obtained for an operating range of $0-100$ psi for pressure sensitivity.
\end{abstract}

Keywords: Piezoresistive / graphene / pressure sensor / COMSOL / simulation

\section{Introduction}

Monitoring human signals is a challenging task when it concerns low signal monitoring. To measure various strain levels of the human physical motion, researchers are focusing on new emerging technology used for the measurement of low signals of the human body [1]. Low signal monitoring is done by utilizing highly sensitive pressure sensors [2]. A high sensitive pressure sensor is achieved by varying the geometrical structure of the sensor and suitable selection of the material.

Graphene is a 2 D material with unique mechanical and electronic properties [3]. The thickness of the graphene varies from mono-layer graphene to the few layer graphene such as $0.34-2.3 \mathrm{~nm}[4,5]$. Graphene has a high modulus of elasticity that makes it a suitable material for various pressure detecting applications [6]. Many researchers work on the fabrication of sensitive graphene pressure sensors for monitoring the health of human beings. Nie et al. [7] proposed a flexible strain sensor by adhering graphene mesh film on liquid crystal polymer substrate to detect the minor input displacement in the human body. Saha et al. [8] propose a wearable sensor fabricated from reduced

\footnotetext{
* e-mail: meetunag@gmail.com
}

graphene oxide patterned paper substrate for investigating the bending and folding angles to detect the motion of the knee, wrists, and fingers. Yang et al. [9] proposed a graphene-based skin line real-time sensor for pulse wave sensing by creating an optimal balance between sensor linearity and sensitivity and reveals that graphene woven fabric, as a sensing element which is responsible for the enhancement in the sensitivity of the sensor. Furthermore, the effect of substrate stiffness with graphene is analyzed in terms of sensitivity and linearity which overall helps in device designing and performance optimization. Chun et al. [10] propose a graphene pressure sensor for tactile sensing using two separated single-layer graphene on a flexible substrate. For low-pressure measurement, a sensitivity of $-0.24 \mathrm{kPa}^{-1}$ and for high-pressure sensitivity of $0.039 \mathrm{kPa}^{-1}$ is achieved. Rinaldi et al. [11] discuss the Multilayer graphene pressure sensor with a sensitivity of $0.23 \mathrm{kPa}^{-1}$ for detecting the compressive stresses as low as $10 \mathrm{kPa}$ for an applied pressure of $70 \mathrm{kPa}$. Inoue et al. [12] propose an inline pressure sensor based on polydimethylsiloxane (PDMS) microfluidic tube integrated with graphene sheets. Inside pressure was monitored by the changes in electrical resistance of the graphene sheet. Chun et al. [13] demonstrate high sensitive tactile sensor of threedimensional interconnected reduced graphene oxide flakes for monitoring the motions of muscles, motions of finger 
and elbow, dynamic pressure induced by an artificial fingerprint structure. A sensitivity of $40.8 \mathrm{kPa}^{-1}$ is achieved for low pressure generated by muscles movement. Huang et al. [14] proposed a hybrid method for enhancing the sensitivity of the pressure sensor.

It is seen that graphene pressure sensors show better results when used for monitoring the pressure sensing of the human body. Considering the geometrical variation in the structure of the piezoresistors, researchers are emphasizing diaphragm geometry and a little bit on the structure of the piezoresistors. Kumar et al. [15] design and simulate piezoresistive pressure sensors to improve the performance of the sensor. Simulation is carried out to investigate the effect of the geometry of the diaphragm, shape, and location of the piezo resistors. Meti et al. [16] designed a piezoresistive pressure sensor by using n-type silicon and p-type meander-shaped piezoresistor. A different structure is simulated using COMSOL multiphysics by varying the length of the piezoresistor to find the best configuration for high sensitivity and linearity. Results reveal that $50 \mu \mathrm{m}$ length of piezoresistors is found to have the best sensitivity. Hayati et al. [17] proposed piezoresistive pressure sensor of hairpin shaped structure. The simulation was carried out using COMSOL multiphysics for square and circular diaphragms for an operating range of $0-10$ bar. The proposed model is analysed for high sensitivity and low nonlinearity.

Even for the fabrication of the graphene pressure sensor involves the compatibility of sticking property of graphene to the oxidized silicon wafer [18]. Graphene due to its strong adhesion property to $\mathrm{SiO}_{2}$, silicon substrate is recommended for the fabrication of the graphene pressure sensor. Qu et al. reported polysilicon pressure sensor and achieve a sensitivity of $0.12 \mathrm{mv} / \mathrm{psi}$ [19]. Polysilicon is said to be suitable material for piezoresistors for sensor operated at room temperature [20]. However, at higher temperature a reduction in sensitivity is noticed [21]. Many researchers exploring the graphene due to its high temperature compatibility at higher temperature also.

As per the discussion of the literature review, it is found that a suitable geometrical structure and selection of proper material can enhance the sensitivity of the pressure sensor utilized for health care monitoring. Considering the variation in the geometrical structure of piezoresistors, limited research is carried out to investigate the shape of the piezoresistors to accommodate the maximum strain area.

In this paper, a new geometry of piezoresistor is discussed that covers the complete area having the maximum strain at the edges for the rigidly clamped diaphragm. With this new geometry of zig-zag shaped piezoresistor, the induced stress in the terms of strain at the edges is covered completely by the piezoresistors to get the output electrical voltage with the help of wheat stone bridge configuration. This placement of piezoresistor affects the performance of the pressure sensor. The operating Pressure range is taken as $0-100$ psi that covers a wide area of application related to health care, automobile sector, etc.

\section{Theory and methodology}

Piezoresistor pressure sensor works on wheat stone bridge configuration having two important parts such as substrate and the sensing element [21]. In this paper silicon is taken as a substrate material which is designed in the form of diaphragm. Due to applied pressure, deformation is induced in the diaphragm which created strain at the edge of the rigid diaphragm [21]. The piezoresistor are placed in zig-zag format to cover the maximum stress areas to convert the change in strain with the help of bridge to the electrical output voltage.

Resistance of the piezoresistor is given by

$$
R=\rho \frac{L}{A}
$$

where $\rho$ the resistivity of the sensing material, $L$ is the length of the piezoresistor, $A$ is the cross-sectional area.

On the application of force, the displacement of the diaphragm at the center is given by

$$
D=0.0151\left(1-\theta^{2}\right) \frac{P a^{4}}{E h^{3}}
$$

where " $P$ " is the pressure, " $a$ " is the side length of square diaphragm, " $h$ " is the thickness of the diaphragm, " $v$ " is the poisons ratio.

Placement of piezoresistors are done to cover the effective strain area at the edges of the rigid diaphragm. Two piezoresistors are placed at the center of the diaphragm to experience the stress at the center also. Two resistors are placed in the center and other are placed at the edges of the diaphragm to measure the tensile and compressive stress. This placement of piezo resistors helps in temperature compensation [1]. COMSOL Multiphysics software is a general-purpose platform for engineering modelling and simulations [22]. The software platform provides an easy way to couple the interactions and effects of different types of physics on a particular application. It has some sub modules that have in built physics functions that can be used to simulate a multitude of effects onto a model. In this study COMSOL Multiphysics 5.3a version for all the simulations [22].

The modules used for the simulation of the pressure sensor is MEMS module and heat transfer module, and for the resistor, the AC/DC module is used. Inside the MEMS module the piezoresistivity sub module is used to characterize the resistance changes with pressure for the piezoresistors. The piezoresistivity is a couple of two physics module, solid mechanics and electric currents.

\subsection{Solid mechanics physics}

The solid mechanics module is used to analyse and simulate the stresses generated on the model due to external pressure. This module also simulates the displacement or deformation of the membrane due to the pressure applied. 


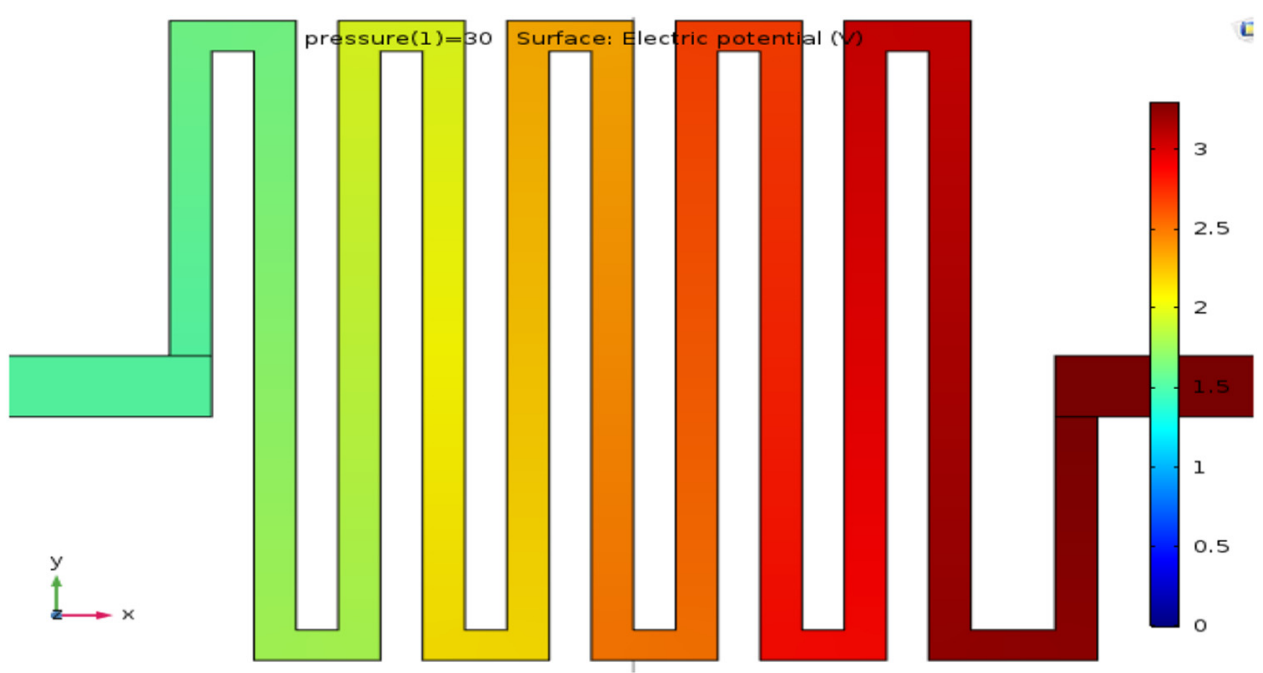

Fig. 1. Shape of piezoresistor.

These stresses and changes in the shape of the diaphragm are used to find the changes in the current across the piezoresistors, by coupling with the electric currents sub module.

\subsection{Electric currents physics}

The electric currents physics in the AC/DC module is used to analyse the currents, potential and power changes happening across a model. The model can also be used to simulate the different loss stresses generated across an electrical component.

\subsection{Heat transfer physics}

Heat transfer physics is added in this simulation, because during simulation the properties of the material change due to the variation in the temperature. Heat transfer module contains a lot of boundary conditions that allow the user to analyse heat transfer by convection, conduction, and radiation. The Heat Transfer physics includes a comprehensive set of features for investigating thermal designs and effects of heat loads. However, temperature is kept constant at room temperature for the analysis of this model. Following is the sequence for modelling the piezoresistive pressure sensor in comsol.

- Define global parameters.

- Pressure.

- Temperature.

- Define components of modelling that includes definition.

- Definition includes: Declaration of boundary probes, metal lines, upper and lower membrane, substrate description.

- Geometry construction by using different work-plane for upper and lower layer.

- Material selection.

- Add physics.

- Meshing.

- Add study.

- Analysis of results.

\subsection{Modelling and simulation}

Performance of piezoresistors are determined by the properties of the substrate and sensing material, placement of the piezoresistor [23,24]. Moreover, dimensions of the piezoresistors and thickness of the square diaphragm effects the performance of the pressure sensor by improving the yield $[25,26]$. In this work, zig-zag shaped piezoresistor is selected to cover the maximum strain at the square diaphragm as shown in Figure 1.

The induced stress is maximum at the edges and minimum at the center of the diaphragm [27]. Diaphragm thickness is chosen to be $50 \mu \mathrm{m}$ for designing of the diaphragm [1]. Boundary conditions with extremely fine meshing are utilized for the simulation of the model. Output voltage is noticed due to the change in resistance of piezoresistor with the help of wheat stone bridge configuration. Figure 2 shows the total displacement. Maximum displacement of the diaphragm is noticed at the center of the diaphragm which is represented as red color. Drop in displacement is noticed as one moves from center to the edges of the rigid diaphragm. The piezoresistors are placed at the center and at the edges of the diaphragm as shown in Figure 2.

\section{Result and discussion}

In this section, the simulated results are analyzed to achieve the output pressure sensitivity. It is noticed that by introducing the zig-zag shaped piezoresistor, the sensitivity of the graphene pressure is achieved as $4.20 \mathrm{mV} / \mathrm{psi}$ for the operating range of $0-100$ psi. Moreover optimization of diaphragm thickness is necessary to increase the sensitivity of the pressure sensor. Thinner diaphragm shows high sensitivity as compared to the thicker diaphragm, but very thin diaphragm causes non linearity in the output results that somehow reduced the sensitivity as non-linearity is added to the output results result that degrades the performance of the pressure sensor. Guan et al. [28] also supports the optimization of the diaphragm thickness. 
Surface: Electric potential (V) $0^{0}$

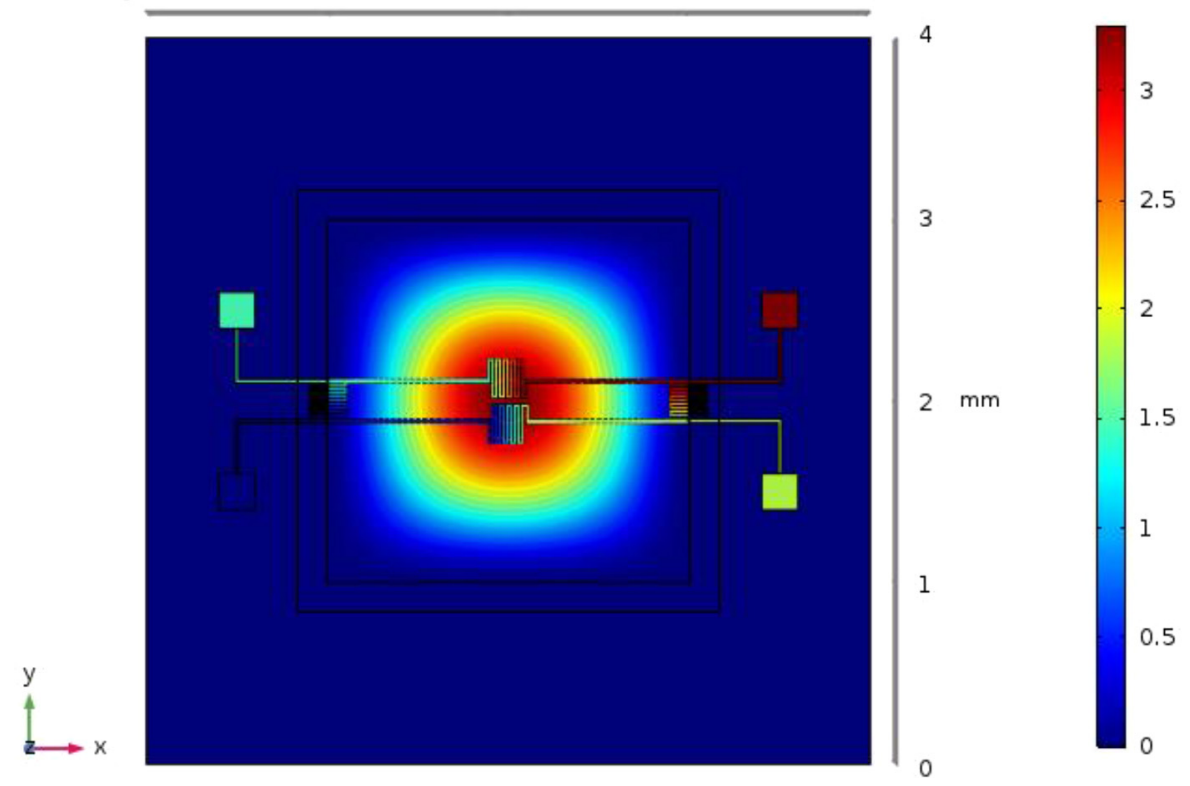

Fig. 2. Simulated model of pressure sensor: total displacement is in mm range at the applied pressure of 100 psi.

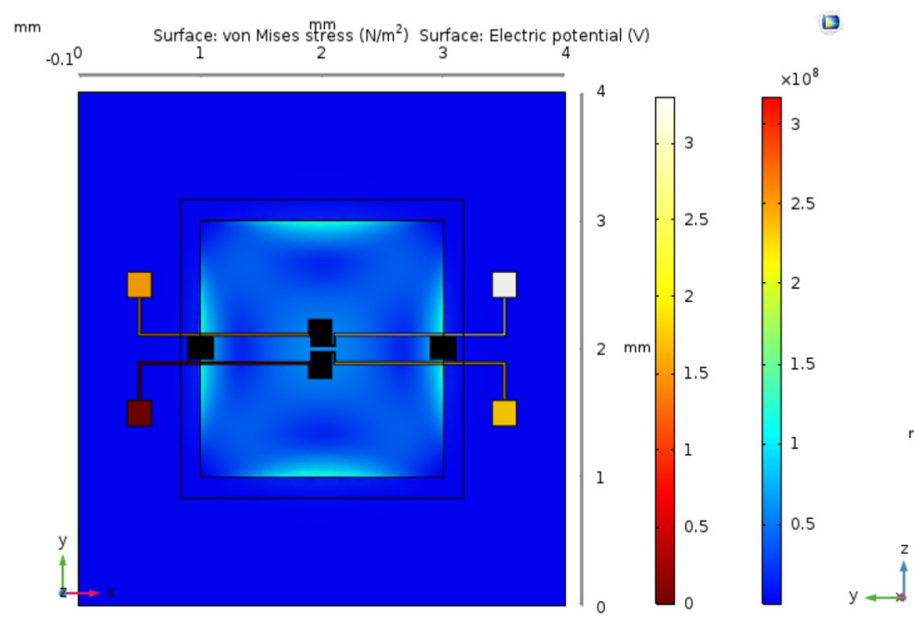

a

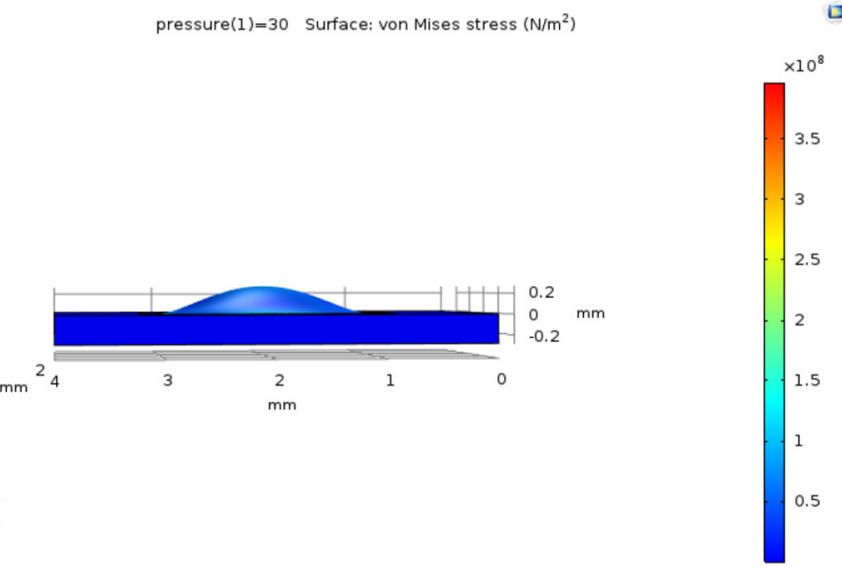

Fig. 3. (a) Top view of the simulated model graphene pressure sensor. (b) Side view of the graphene pressure sensor.

Dimensions of the piezoresistor plays an important role in enhancing the sensitivity of the pressure sensor but again very large length is not entertained for fabrication of the pressure sensor as graphene is used as s sensing element with mono layer thickness that limits the dimensions of the piezoresistors. Increasing the length of the piezoresistors beyond limits decreases the sensitivity $[29,30]$. Placement of the piezoresistors also affects the sensitivity of the pressure sensor $[31,32]$.

However, the proposed simulated model of graphene pressure sensor has various advantages on the other sensing material due to the unique properties of graphene and temperature compatibility at the higher temperature. This modeled design can be utilized for the fabrication of graphene pressure sensor for health care devices as this zig-zag shaped piezoresistor covers the maximum strain area which makes its more suitable for medical filed where more sensitivity is required to measure the low pressure.

Figure $3 \mathrm{a}$ and $\mathrm{b}$ shows the top and side view of the simulated model with zig-zag shaped piezoresistors.

On the application of applied pressure ranges from 0 to 100 psi, output voltage is calculated by COMSOL Multiphysics by applying the boundary conditions to the model. It is noticed in Figure 4 that an approx. linear relationship is noticed between the applied pressure and the output voltage. The results shows that initially the relation follows slightly polynomial relation but as the pressure is increased that curve shown in Figure 4 shows linear relation between input and the output voltage. 


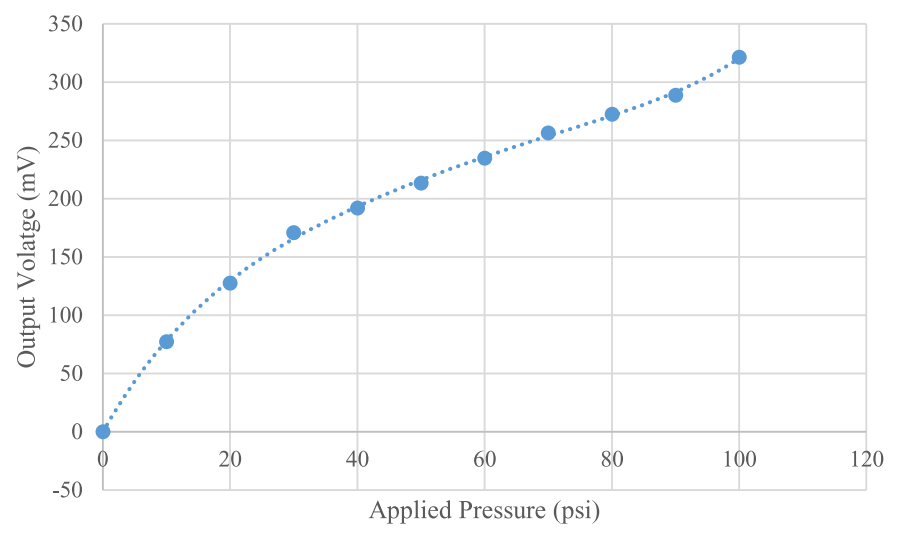

Fig. 4. Output characteristic of graphene pressure.

\section{Conclusion}

In this work, zig-zag shaped piezoresistors are designed to cover the maximum strain region at the edges of the diaphragm. From this simulated geometry, a sensitivity of $4.20 \mathrm{mV} / \mathrm{psi}$ is achieved for an operating range of $0-100 \mathrm{psi}$ with the help of wheat stone bridge configuration. From the simulation, it is noticed that the combination of silicon as a substrate material and graphene as a sensing material enhances the sensitivity of the sensor within a wide range that covers the working range of medical pressure sensor, health care sensor, etc. The simulation is carried out using COMSOL Multiphysics 5.3 a version. From Figure 4, it is clear that as the applied pressure increases, a noticeable change is seen in the output in the form of electrical voltage. Maximum deflection is noticed at the center of the square diaphragm. Nag et al. [21] simulated a graphene pressure sensor for a single turn of piezoresistor and achieved the maximum sensitivity of $4.00 \mathrm{mV} / \mathrm{psi}$. At room temperature, a sensitivity of $3.98 \mathrm{mV} / \mathrm{psi}$ is achieved. It is clear that by increasing the surface area for piezoresistors, sensitivity is improved by $0.22 \mathrm{mV} / \mathrm{psi}$. This sensor can be used in various applications such as in health care monitoring devices and the automobile sector [32-34]. For future enhancement, optimization is carried out to enhance the sensitivity of the pressure sensor. Even during the fabrication of graphene pressure sensor, effective transfer of CVD graphene on the oxidized silicon wafer adds to the sensitivity of the pressure sensor.

Conflicts of interest. The authors declare that they have no known competing financial interests or personal relationships that could have appeared to influence the work reported in this paper.

\section{References}

1. M. Nag, J. Singh, A. Kumar, K. Singh, A high sensitive graphene piezoresistive MEMS pressure sensor by integration of rod beams in silicon diaphragm for low pressure measurement application, Microsyst. Technolog. 26, 2971-2976 (2020)
2. M. Nag, M. Lamba, K. Singh, A. Kumar, Modelling and simulation of MEMS graphene pressure sensor for healthcare devices, in Proceedings of International Conference in Mechanical and Energy Technology (Springer, Singapore, 2020), pp. 607-612

3. Y. Pang, Z. Yang, Y. Yang, X. Wu, Y. Yang, T.L. Ren, Graphene based wearable sensors for healthcare, in 2019 International Conference on IC Design and Technology (ICICDT) (IEEE, 2019), pp. 1-4

4. Y. Shi, L. Ye, A.H. Zehri, N. Logothetis, P. Su, N. Wang, J. Liu, Fabrication and characterization of graphene based film, in 2017 IMAPS Nordic Conference on Microelectronics Packaging (NordPac) (IEEE, 2017), pp. 162-166

5. H. Tian, Y. Shu, X.F. Wang, M.A. Mohammad, Z. Bie, Q.Y. Xie et al., A graphene-based resistive pressure sensor with record-high sensitivity in a wide pressure range, Sci. Rep. 5, 1-6 (2015)

6. M.A. Shazni, M.W. Lee, H.W. Lee, Highly-sensitive graphene-based flexible pressure sensor platform, Sains Malaysiana 46, 1155-1161 (2017)

7. M. Nie, Y.H. Xia, H.S. Yang, A flexible and highly sensitive graphene-based strain sensor for structural health monitoring, Cluster Comput. 22, 8217-8224 (2019)

8. B. Saha, S. Baek, J. Lee, Highly sensitive bendable and foldable paper sensors based on reduced graphene oxide, ACS Appl. Mater. Interfaces 9, 4658-4666 (2017)

9. T. Yang, X. Jiang, Y. Zhong, X. Zhao, S. Lin, A wearable and highly sensitive graphene strain sensor for precise home-based pulse wave monitoring, ACS Sensors 2, 967-974 (2017)

10. S. Chun, Y. Kim, H.S. Oh, G. Bae, W. Park, A highly sensitive pressure sensor using a double-layered graphene structure for tactile sensing, Nanoscale 7, 11652-11659 (2015)

11. A. Rinaldi, A. Tamburrano, M. Fortunato, M.S. Sarto, A flexible and highly sensitive pressure sensor based on a PDMS foam coated with graphene nanoplatelets, Sensors 16, 2148 (2016)

12. N. Inoue, H. Onoe, Graphene-based inline pressure sensor integrated with microfluidic elastic tube, J. Micromech. Microeng. 28, 014001 (2017)

13. S. Chun, H. Jung, Y. Choi, G. Bae, J.P. Kil, W. Park, A tactile sensor using a graphene film formed by the reduced graphene oxide flakes and its detection of surface morphology, Carbon 94, 982-987 (2015)

14. C.B. Huang, S. Witomska, A. Aliprandi, M.A. Stoeckel, M. Bonini, A. Ciesielski, P. Samorì, Molecule-graphene hybrid materials with tunable mechanoresponse: highly sensitive pressure sensors for health monitoring, Adv. Mater. 31, 1804600 (2019)

15. U.S. Kumar, N.J. Babu, Design and simulation of MEMS Piezoresistive Pressure Sensor to Improve the sensitivity, Int. J. Innov. Res. Electr. Electr. Instrum. Control Eng. 3 (2015)

16. S. Meti, K.B. Balavalad, A.C. Katageri, B.G. Sheeparamatti, Sensitivity enhancement of piezoresistive pressure sensor with meander shape piezoresistor, in 2016 International Conference on Energy Efficient Technologies for Sustainability (ICEETS) (IEEE, 2016), pp. 890-895

17. M. Hayati, M. Fathipour, H.S. Vaziri, Design and analysis of hairpin piezoresistive pressure sensor with improved linearity using square and circular diaphragms, Micro Nano Lett. 13, 1046-1051 (2018) 
18. J. Ou, J. Wang, S. Liu, B. Mu, J. Ren, H. Wang, S. Yang, Tribology study of reduced graphene oxide sheets on silicon substrate synthesized via covalent assembly, Langmuir 26, 15830-15836 (2010)

19. Q. Hongwei, Y. Suying, Z. Rong, M. Ganru, Z. Weixin, M. Xiaoqiang, L. Lei, Polysilicon piezoresistive pressure sensor and its temperature compensation, in 1998 5th International Conference on Solid-State and Integrated Circuit Technology. Proceedings (Cat. No. 98EX105) (IEEE, 1998), pp. 914-916

20. M.H.M. Khir, P. Qu, H. Qu, A low-cost CMOS-MEMS piezoresistive accelerometer with large proof mass, Sensors 11, 7892-7907 (2011)

21. M. Nag, J. Singh, A. Kumar, P.A. Alvi, K. Singh, Sensitivity enhancement and temperature compatibility of graphene piezoresistive MEMS pressure sensor, Microsyst. Technolog. 25, 3977-3982 (2019)

22. W.B. Zimmerman, Introduction to COMSOL multiphysics, in Multiphysics Modeling with Finite Element Methods Citation Key: Zimmerman 2006 (World Scientific Publishing Company, 2006), pp. 1-26

23. A.A. Barlian, W.T. Park, J.R. Mallon, A.J. Rastegar, B.L. Pruitt, Semiconductor piezoresistance for Microsystems, Proc. IEEE 97, 513-552 (2009)

24. S.T.A. Hamdani, A. Fernando, The application of a piezoresistive cardiorespiratory sensor system in an automobile safety belt, Sensors 15, 7742-7753 (2015)

25. K.Y. Madhavi, M. Krishna, C.C. Murthy, Effect of diaphragm geometry and piezoresistor dimensions on the sensitivity of a piezoresistive micropressure sensor using finite element analysis, IJESE 1 (2013)

26. J. Akhtar, B.B. Dixit, B.D. Pant, V.P. Deshwal, Polysilicon piezoresistive pressure sensors based on MEMS technology, IETE J. Res. 49, 365-377 (2003)
27. N. Barakat, A. Plotkowski, H. Jiao, Design and Computational analysis of Diaphragm Based Piezoresistive Pressure Sensors for Integration into Undergraduate Curriculum (2011)

28. T. Guan, F. Yang, W. Wang, X. Huang, B. Jiang, D. Zhang, The design and analysis of piezoresistive shuriken-structured diaphragm micro-pressure sensors, J. Microelectromech. Syst. 26, 206-214 (2016)

29. J.C. Doll, S.J. Park, B.L. Pruitt, Design optimization of piezoresistive cantilevers for force sensing in air and water, J. Appl. Phys. 106, 064310 (2009)

30. J. Sosa, J.A. Montiel-Nelson, R. Pulido, J.C. GarciaMontesdeoca, Design and optimization of a low power pressure sensor for wireless biomedical applications, J. Sens. 2015 (2015)

31. S.K. Jindal, S.P. Magam, M. Shaklya, Analytical modeling and simulation of MEMS piezoresistive pressure sensors with a square silicon carbide diaphragm as the primary sensing element under different loading conditions, J. Comput. Electr. 17, 1780-1789 (2018)

32. A.E. Kubba, A. Hasson, A.I. Kubba, G. Hall, A microcapacitive pressure sensor design and modelling, J. Sens. Sens. Syst. 5, 95-112 (2016)

33. M. Nag, M. Lamba, K. Singh, A. Kumar, Modelling and simulation of MEMS graphene pressure sensor for healthcare devices, in Proceedings of International Conference in Mechanical and Energy Technology (Springer, Singapore, 2020), pp. 607-612

34. M. Nag, A. Kumar, K. Singh, B. Pratap, Graphene based flexible piezoresistive pressure sensor for electric vehicles applications, in AIP Conference Proceedings (AIP Publishing LLC, 2020), Vol. 2294, p. 020009

Cite this article as: Meetu Nag, Ajay Kumar, Bhanu Pratap, A novel graphene pressure sensor with zig-zag shaped piezoresistors for maximum strain coverage for enhancing the sensitivity of the pressure sensor, Int. J. Simul. Multidisci. Des. Optim. 12, 14 $(2021)$ 